\title{
Blüten- und Bestäubungsvielfalt bei Sinningia (Gesneriaceae)
}

\author{
Alain Chautems, Mathieu Perret \& Anton Weber
}

\begin{abstract}
The family Gesneriaceae exhibits an enormous diversity regarding flower form, colour, size and pollination syndromes. Of special interest are cases in which the evolutionary diversification of flowers and adaptation to particular animal groups took place within a genus or group of closely related genera. From the evolutionary viewpoint this means that diversification occurred in a remarkably short time. Here the neotropical complex of the genera Sinningia-Paliavana-Vanhouttea (Sinningia s. 1.) is treated which could serve as a text-book example for rapid adaptation to different pollinators. The spectrum includes various bee groups (euglossine and other bees), butterflies, moths, birds (hummingbirds) and bats. Molecular-phylogenetic studies demonstrate that the adaptations partly evolved several times and independently.
\end{abstract}

\section{Zusammenfassung}

Die Familie der Gesneriaceae weist hinsichtlich Blütenformen, -farben, -größen und Bestäubungssyndromen eine enorme Vielfalt auf. Besonders interessant sind jene Fälle, bei denen sich innerhalb einer Gattung oder einer engen Verwandtschaftsgruppe verschiedene Bestäubungssyndrome und Anpassungen an bestimmte Tiergruppen herausdifferenziert haben - evolutionär gesehen, also in einer relativ kurzen Zeit. Geradezu als Lehrbuchbeispiel kann diesbezüglich der neotropische Gattungskomplex Sinningia-Paliavana-Vanhouttea (Sinningia s. 1.) gelten, in dem sich die Blüten in spezifischer Weise an die Bestäubung durch verschiedene Bienengruppen (Pracht- und andere Bienen), Schmetterlinge (Tagfalter), Nachtfalter, Vögel (Kolibris) und Fledermäuse angepasst haben. Molekular-phylogenetische Studien zeigen, dass die genannten Syndrome z. T. mehrfach und voneinander unabhängig entstanden sind.

\section{Einleitung}

Die Familie der Gesneriaceae ist hierzulande hauptsächlich durch zwei Zimmerpflanzen bekannt, nämlich das Usambara-Veilchen (Streptocarpus ionanthus, besser bekannt unter dem alten Namen Saintpaulia ionantha), und die sogenannten „Gloxinien“ (Sinningia speciosa, mit zahlreichen gärtnerischen Varianten). Die letztere Gattung Sinningia steht im Mittelpunkt des vor-

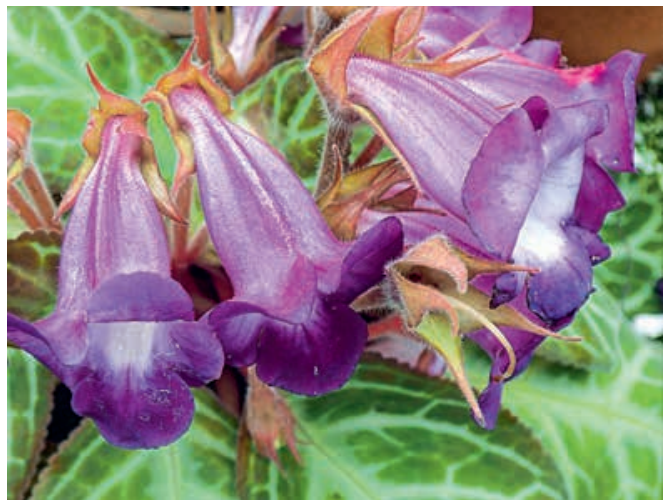

Abb. 1: Sinningia speciosa, Wildform in Brasilien (Rio de Janeiro, nahe Campos dos Goytacazes, Serra da Vista). Die Art bildet den Ausgangpunkt für zahlreiche gärtnerische Varianten und Zuchtformen, die unter dem irreführenden Namen „Gloxinien“ geführt werden. (Foto: A. Chautems) liegenden Beitrages, in dem es um die erstaunliche Blütenvielfalt und die verschiedenen Bestäubungsmuster geht, die sich im Laufe der Evolution in dieser Gattung entwickelt haben.

Die Gesneriaceen sind eine hauptsächlich tropisch-subtropisch verbreitete Familie, die bis auf wenige Selbstbestäuber tierblütig ist. Außer Käfern und nicht-fliegenden Säugetieren gibt es kaum eine blütenökologisch relevante Tiergruppe, die von Vertretern dieser Familie nicht in den Dienst der Pollenübertragung gestellt wurde. Die ungeheure Vielfalt an Blütenformen, -farben und -größen muss im $\mathrm{Zu}$ sammenhang mit der Bestäubung gesehen werden. Gleichartige oder ähnliche Merkmalsmuster in den Blüten ergeben die sog. Bestäubungssyndrome und diese deuten auf die Bestäubung durch spezifische Tierklassen hin: Insekten wie Käfer (Cantharophilie), Bienen (Melittophilie), Fliegen (Myiophilie), Tagfalter (Psychophilie), Nachtfalter (Sphingophilie und Phalenophilie) oder Wirbeltiere wie Vögel (Ornithophilie), Fledermäuse (Chiropterophilie) oder nicht-fliegende Säugetiere (Therophilie), um die wichtigsten zu nennen. Die Voraussagekraft der Bestäubungssyndrome ist allerdings nicht hundertprozentig. Es gibt immer wieder Überraschungen 
bzw. Feinschattierungen der Syndrome, die in die Irre führen können. Den Schlussfolgerungen aus der Blütenmorphologie müssen somit Beobachtungen und Studien an den Naturstandorten folgen.

Dass sich in einer so großen Familie wie den Gesneriaceen (ca. 150 Gattungen und etwa 3700 Arten) im Laufe der Evolution verschiedene Bestäubungssyndrome herausgebildet haben, ist nicht weiter überraschend. Interessant sind jene Fälle, bei denen sich verschiedene Syndrome in einer engen Verwandtschaftsgruppe, z. B. in einer Gattung, entwickelt haben. Es gibt eine Reihe von Gattungen, bei denen mehr als ein einziges Bestäubungssyndrom zu finden ist, z. B. bei Achimenes, Didymocarpus, Kohleria, Streptocarpus, Oreocharis, Petrocodon und Sinningia.

Hier soll die neotropische Gattung Sinningia bzw. der ineinander verschränkte Gattungskomplex Sinningia-Paliavana-Vanhouttea (Sinningia s. l., siehe unten) herausgegriffen werden. Hier zeigt sich die größte Vielfalt an Syndromen innerhalb der Gesneriaceae, die geradezu als Lehrbuchbeispiel für eine blütenökologische Differenzierung innerhalb einer Gattung dienen kann. Mit Ausnahme von Parfümblumen (die gibt es nur in der Gattung Gloxinia, Vogel 1966, siehe dazu Weber \& Gerlach 2018, Martel et al. 2019 und darin zitierte Literatur) und von Fliegenblumen (einige Arten von Streptocarpus, Beobachtungen von J. Manning, zitiert in РотGIETER \& EDWARDS 2005) sind hier praktisch alle morphologisch-funktionellen Blütentypen und spezialisierte Bestäubergruppen der Gesneriaceae vertreten.

\section{Allgemeines}

Die Gattung Sinningia wurde 1825 von dem deutschen Arzt und Naturforscher NeEs von EsENBECK (1776-1851) beschrieben und nach dem damaligen Gärtner und späteren Leiter des Königlich preußischen Botanischen Gartens Bonn, Wilhelm Sinning (1792-1884), benannt. Näheres über diese beiden interessanten Personen ist Barthlotт (1990) zu entnehmen. Die als erste beschriebene Art (Typusart) war Sinningia helleri (Abb. 5). Sie war lange Zeit verschollen und wur- de erst 2015 in Brasilien nahe der Kleinstadt Miguel Pereira (120 km entfernt von Rio de Janeiro) wiederentdeckt.

Sinningia umfasst nach heutigem Wissen 78 Arten, die Nachbargattungen Paliavana 6 und Vanhouttea 9 Arten. Molekularsystematische Untersuchungen zeigen, dass die Arten von Paliavana und Vanhouttea an verschiedenen Stellen in Sinningia eingebettet sind (Perret et al. 2003, siehe auch Abb. 13). Formal taxonomisch sind diese Arten allerdings bis heute nicht in Sinningia eingegliedert worden. Es soll daher im Folgenden zwischen Sinningia im engeren (s. s.) und im weiteren Sinne (s. l.) unterschieden werden.

Fast alle Arten von Sinningia s. l. kommen im atlantischen Waldgebiet von Brasilien (Ost- und Südbrasilien) vor, nur bei einigen wenigen erstreckt sich das Verbreitungsgebiet vom brasilianischen Küstengebiet nach Nord-Argentinien, Paraguay und, den Anden folgend, bis nach Kolumbien und den westlichen Teil des Amazonasbeckens. Als einzige Art erreicht $S$. incarnata Mittelamerika.

Nicht nur blütenmorphologisch und -ökologisch, sondern auch im Bau des Vegetationskörpers, lässt sich eine enorme Vielfalt beobachten. Das Spektrum reicht von 1-2,5 cm kleinen Zwergpflänzchen mit wenigen Millimeter großen Blüten (S. minima) bis zu $1-1,5 \mathrm{~m}$ hohen Sträuchern (S. gesneriifolia, S. reitzii, S. mauroana). Die meisten Arten von Sinningia s. s. bilden Knollen, die im Extremfall bis zu einem Meter Durchmesser erreichen können. Sie dienen der Wasser- und Nährstoffspeicherung und sind die einzigen Teile der Pflanze, die die Trockenzeiten überdauern können. Nach Einsetzen der Regensaison treiben aus den Knollen Sprosse mit Blättern und Blüten, die nach dem Fruchten wieder absterben. Zwei Arten, S. cooperi (Abb. 3) und $S$. douglasii, wachsen meist epiphytisch, die anderen terrestrisch oder auf Felsen.

\section{Blütenformen, Bestäubungssyndrome und Bestäuber}

Die Abb. 2 zeigt die hauptsächlichen Blütenformen, die in der Gattung Sinningia s. l. anzutreffen sind: Kasten A: Rote, relativ lang- und engröhrige Blü- 


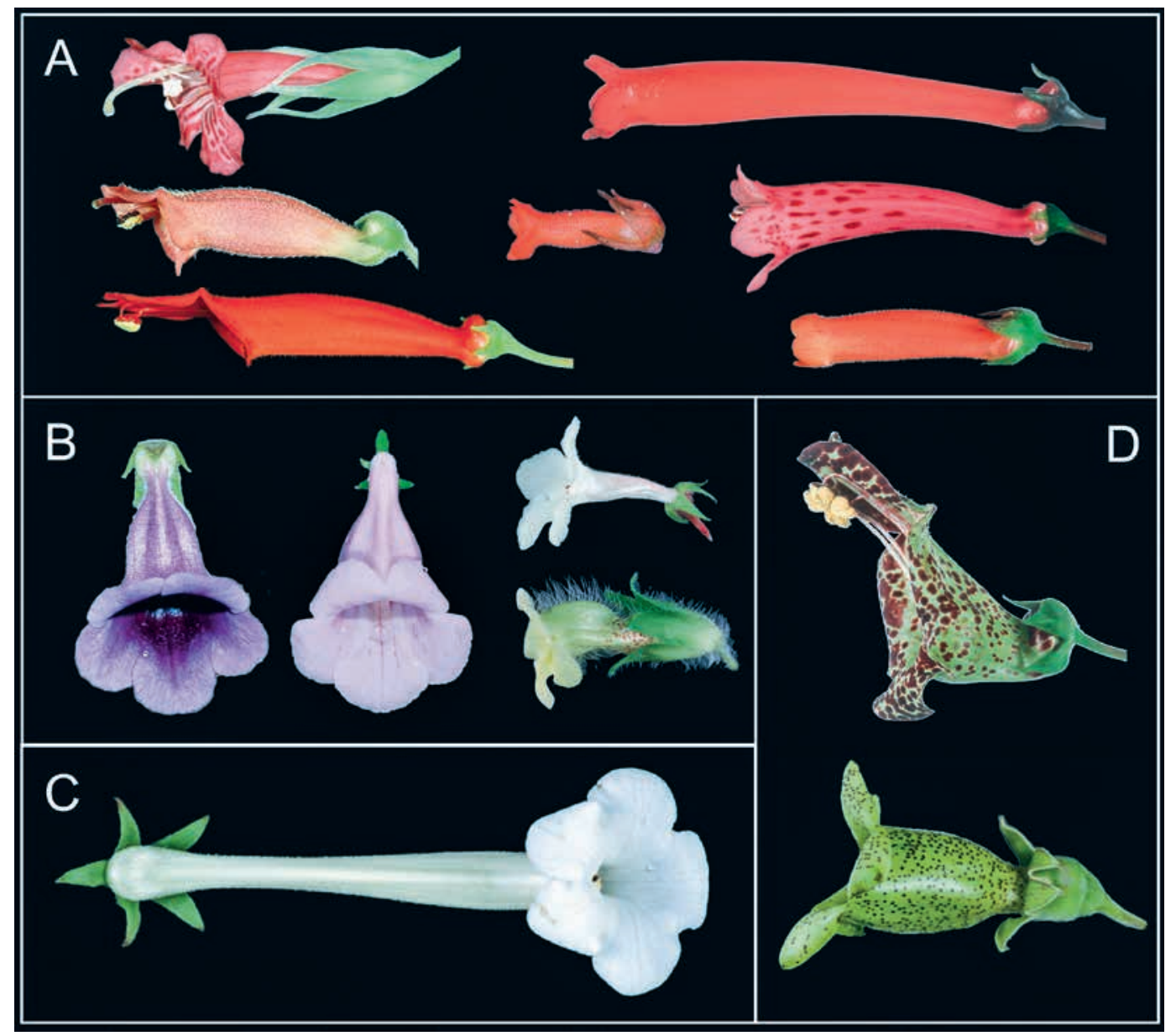

Abb. 2: Blütenvielfalt und Bestäubungssyndrome in der Gattung Sinningia s. 1. Kasten A: Kolibribestäubte Blüten (linke Spalte: Vanhouttea bruggeri, Sinningia elatior, S. bulbosa - alle mit vergrößerter, vorgestreckter Oberlippe); Mitte: S. allagophylla; rechte Spalte: S. gigantifolia, S. douglasii, S. aggregata - alle mit einem \pm radiären Saum mit kleinen, fast gleichen Kronzipfeln). Kasten B: Bienenbestäubte Blüten, links und Mitte: Blüten mit weiter, bauchig-glockiger Krone (Sinningia aghensis, S. eumorpha); rechts: Blüten mit enger Kronröhre (oben: Sinningia schiffneri, unten: S. villosa). Kasten C: Die aller Wahrscheinlichkeit nach von Nachtfaltern (Sphingidae) bestäubte Blüte von S. tubiflora. Kasten D: Die erwiesenermaßen fledermausbestäubten Blüten von Sinningia brasiliensis (oben) und Paliavana prasinata (unten). (Fotos \& Tafelzusammenstellung: M. Perret)

ten. Sie gehören der dominierenden Gruppe der vogelbestäubten Arten an (ca. 50 bzw. zwei Drittel der Arten; bei Vanhouttea sind alle neun Arten vogelblütig, bei Paliavana vermutlich zwei). Blütenmorphologisch zerfallen die Arten in zwei Gruppen: (1) solche mit ausgeprägter und gerade nach vorne gestreckte Oberlippe, während die Unterlippe relativ klein ist, und (2) Blüten mit kleinem, radiärsymmetrischen oder schwach zygomorphen Saum und fast gleich großen Kronzipfeln. Die Blüten des erstgenannten Typs sind die fortschrittlicheren und effizienteren. Unter dem vorgestreckten Dach der Oberlippe liegen die zwei Antherenpaare, die somit punktgenau den Pollen auf den Kopf oder den Schnabel des Kolibris applizieren können. Die Fernanlockung dieser Tiere erfolgt ausschließlich visuell. Die roten Blüten sind weithin sichtbar und attraktiv. Da der Geruchssinn bei fast allen Vögeln unterentwickelt ist, wäre Duftproduktion reine Verschwendung. Als blütenbesuchende Vögel wurden bei drei untersuchten Arten von Sinningia sowie drei Arten von Vanhouttea ausschließlich Kolibris 


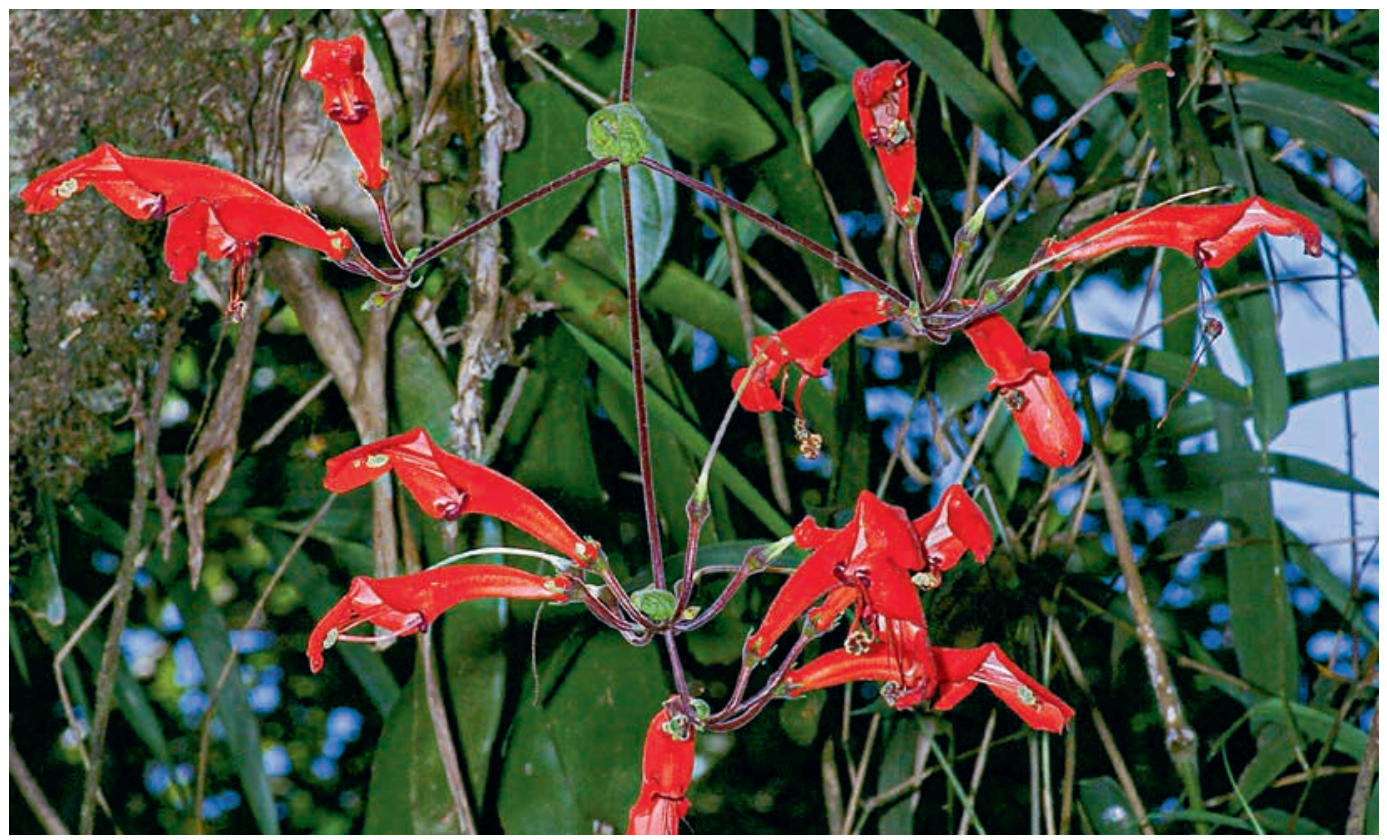

Abb. 3: Sinningia cooperi, ein fakultativer Epiphyt, an seinem Naturstandort in Brasilien (Edo. Sao Paulo, Parque Estadual Serra do Mar, Sta. Virginia, 900 m); die kolibribestäubten Blüten repräsentieren den Typ A, linke Reihe, von Abb. 2.

(Foto: G. Gerlach)

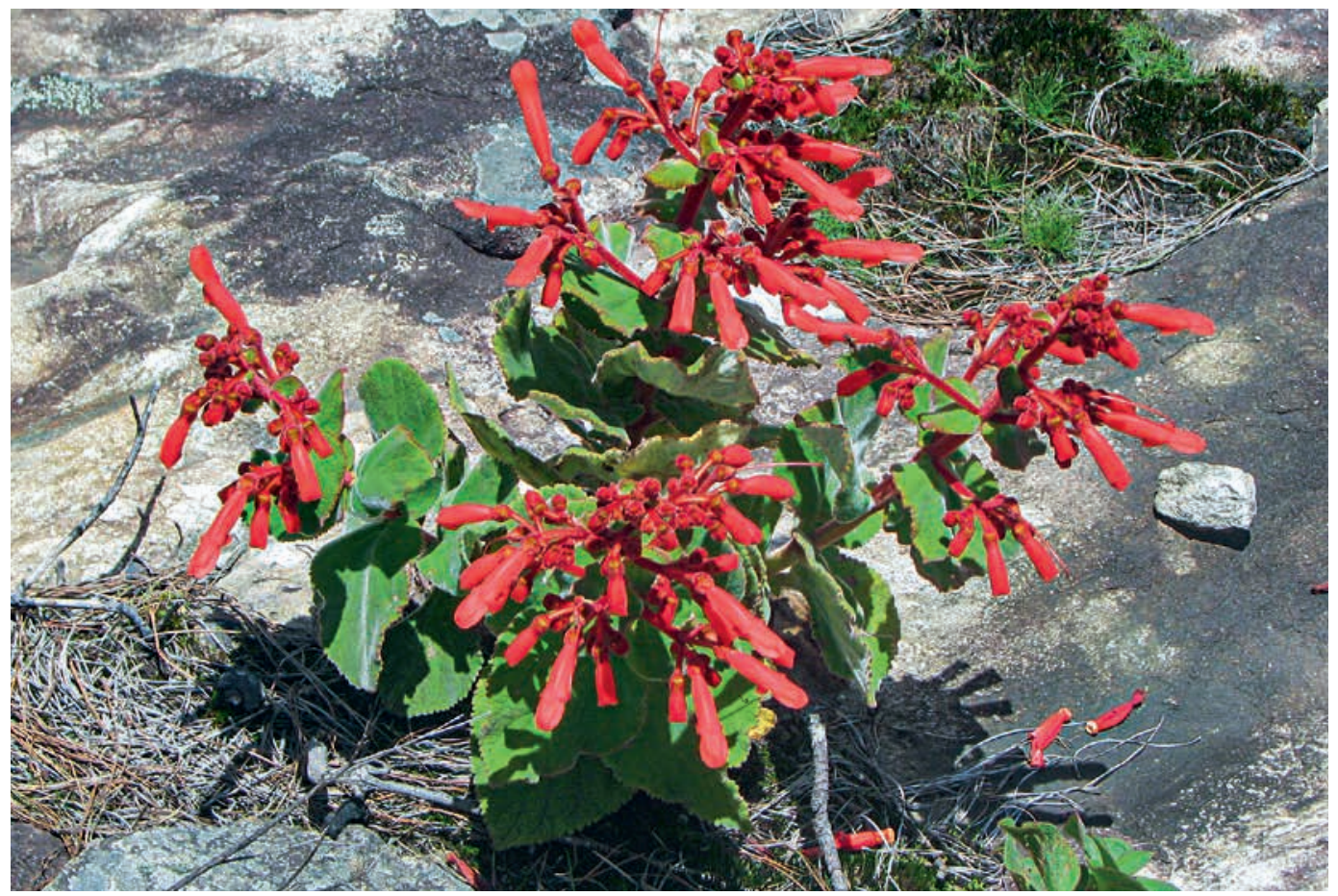

Abb. 4: Sinningia magnifica, eine terrestrische Art, an ihrem Naturstandort in Brasilien (Sao Paulo, Campos do Jordao); die kolibribestäubten Blüten repräsentieren ebenfalls den Typ A, linke Reihe von Abb. 2. (Foto: M. Регхото) 


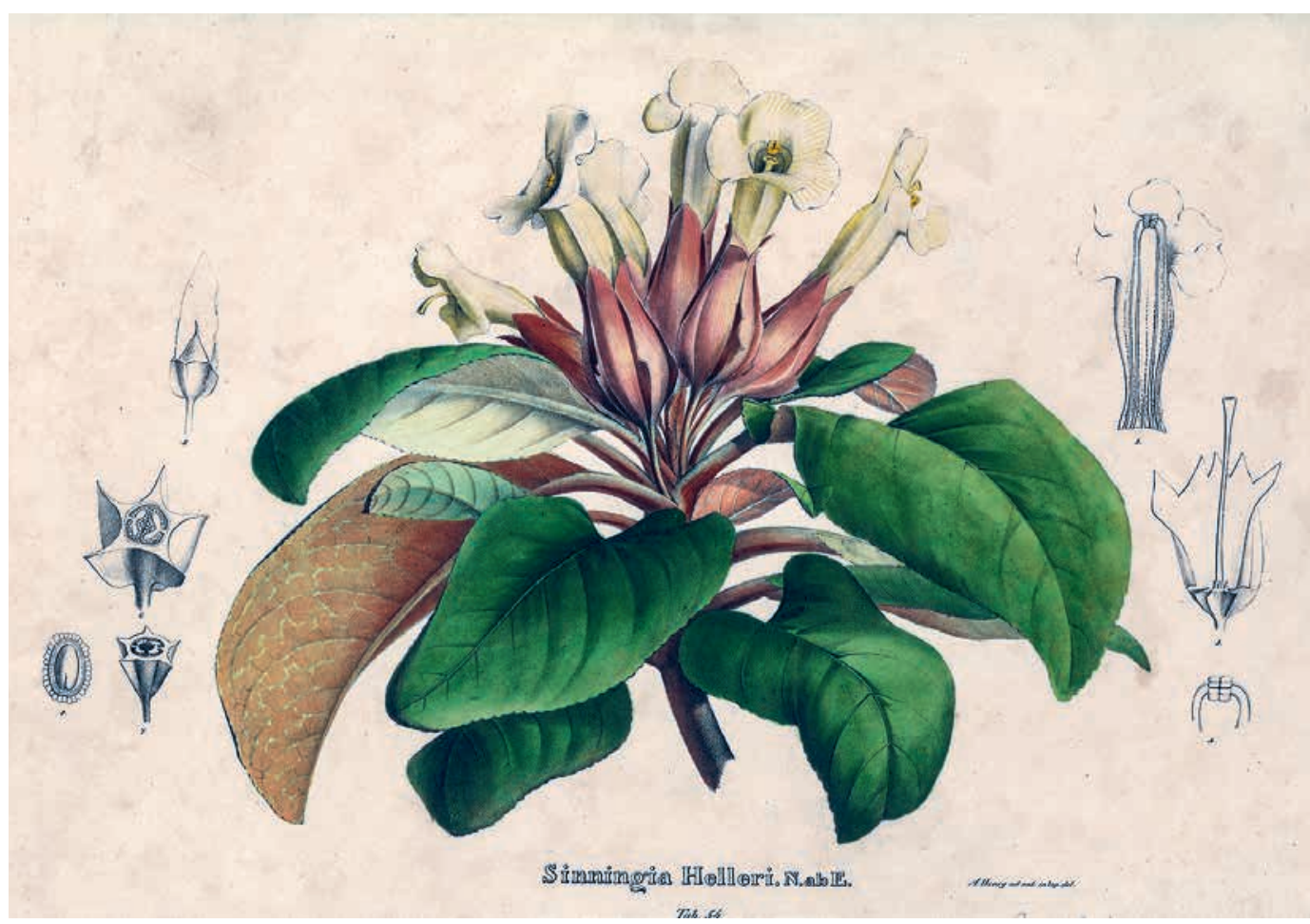

Abb. 5: Sinningia helleri, die Typus-Art von Sinningia - hier in einer historischen Illustration (Esenbeck \& SinNING, Samml. Schönblüh. Gewächse, Tab. 54, 1831). Der Farbkontrast zwischen dem - in natura leuchtend roten - Kelch und der weißen Korolle deutet auf Vogel-(Kolibri-)bestäubung hin. Beobachtungen fehlen.

(Trochilidae: Clytolaema rubricauda, Leucochloris albicollis, Phaethornis eurynome, P. pretrei und Stephanoxis lalandi) identifiziert (SANMARTIN-GaJARDo \& SAZIMA 2005a).

Die zweite große Gruppe weist Bienenblumen auf. Sie sind die phylogenetisch ursprünglichen Vertreter, nicht nur bei Sinningia, sondern bei den neu- wie altweltlichen Gesneriaceen insgesamt (Serrano-Serrano et al. 2017). Auch diese Blüten treten uns in zwei Ausprägungsformen entgegen: (1) Blüten mit weiter, bauchiger oder glockiger Kronröhre. Die Farben variieren von weiß über gelb, blau, blauviolett zu schmutzigrötlich (Abb. 2, Kasten B, links und Mitte). (2) Blüten mit enger Kronröhre und weitem Schlund. Die Farben sind hier vielfach heller (siehe Abb. 2, Kasten B, rechts oben und unten).

Lange Zeit wurden männliche und weibliche Prachtbienen (Euglossinen) als für die einzigen
Bestäuber gehalten (Gynandro-Euglossophilie), doch spielen nach neueren Erkenntnissen auch andere Bienen eine wichtige Rolle. Die bis dato einzige Studie zur Bienenbestäubung von Sinningia wurde von SanMartin-Gajardo \& SaZiMA (2004) vorgelegt. Von den vier untersuchten Arten (S. canastrensis, S. eumorpha, S. schiffneri, S. villosa), wurde nur $S$. villosa ausschließlich von Prachtbienen besucht, die anderen hingegen auch von Hummeln (Bombus morio), Centridinen (Epicharis morio) und Tapinotaspidinen (Trigonopedia ferruginea).

Was die Fledermausbestäubung betrifft, ist bei Sinningia s. s. nur die eine Art Sinningia brasiliensis betroffen (Abb. 2 D, 6, 12). Sie wurde früher als eigene, monotypische Gattung (Lietzia) geführt. Die Blüte ist eine weit offene Rachenblume mit gelblich-grüner Grundfärbung und braun-violetten Flecken. Über den Duft gehen die Meinungen auseinander. Manche empfinden ihn als süßlich- 


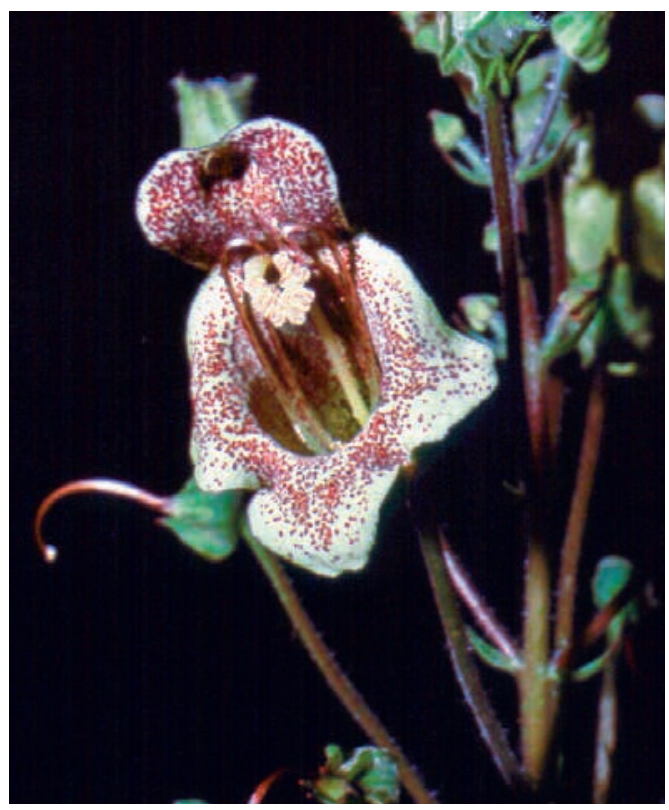

Abb. 6: Sinningia brasiliensis (cult. BG Univ. Wien. Vgl. dazu auch Abb. 2 D und Abb. 12) ist die einzige Art der Gattung Sinningia s. s., die fledermausbestäubte Blüten bildet. Rechnet man Paliavana und Vanhouttea mit ein (Sinningia s. 1.), ist Fledermausblütigkeit mindestens dreimal parallel entstanden (vgl. Abb. 13). (Foto: A. Weber)

honigartig (BogGAN 1966), andere erinnert er an verbranntes Plastik (Perret et al. 2001). Wie SanMartin-Gajardo \& Sazima (2005b) nachgewiesen haben, erfolgt die Bestäubung durch die Geschwänzte Langnasenfledermaus (Anoura caudifer), eine kleine, wendige Blumenfledermaus, die auch als Bestäuber anderer neotropischer Pflanzen bekannt ist. Größe und Form der Blüte und des Fledermauskopfes sind genau aufeinander abgestimmt (Abb. 12). Rechnet man Paliavana und Vanhouttea dazu, so kommen weitere chiropterophile Arten hinzu: Paliavana prasinata, P. plumerioides, $P$. werdermannii und $P$. stapelioides, die drei letzteren allerdings mit Fragezeichen, da das Bestäubungssyndrom nicht so eindeutig ist und keine Beobachtungen vorliegen. Umgekehrt hat sich auch das Spektrum der Fledermäuse erweitert, insbesondere um die Gemeine Blütenfledermaus (Glossophaga soricina), die häufigste Blütenfledermaus in Süd- und Mittelamerika. Im phylogenetischen Diagramm von Abb. 13 kann man sehen, dass die Fledermausblütigkeit bei Sinningia s. 1. mindestens dreimal parallel entstanden ist. Nicht alle wahr-

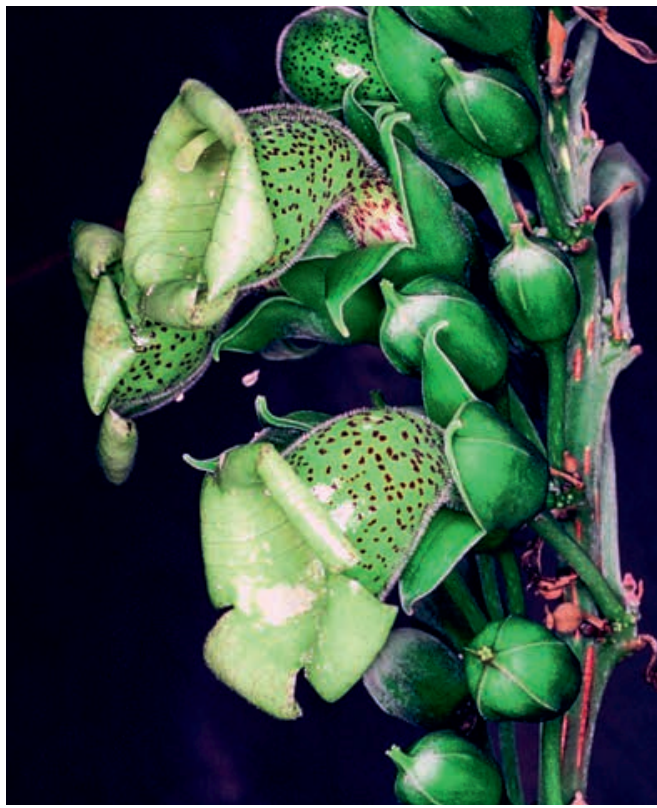

Abb. 7: Paliavana prasinata. in Brasilien (nähere Fundortdetails unbekannt). Die Blüten weisen klar chiropterophile Merkmale auf und werden erwiesenermaßen von Fledermäusen bestäubt. (Foto: S. VoGEL)

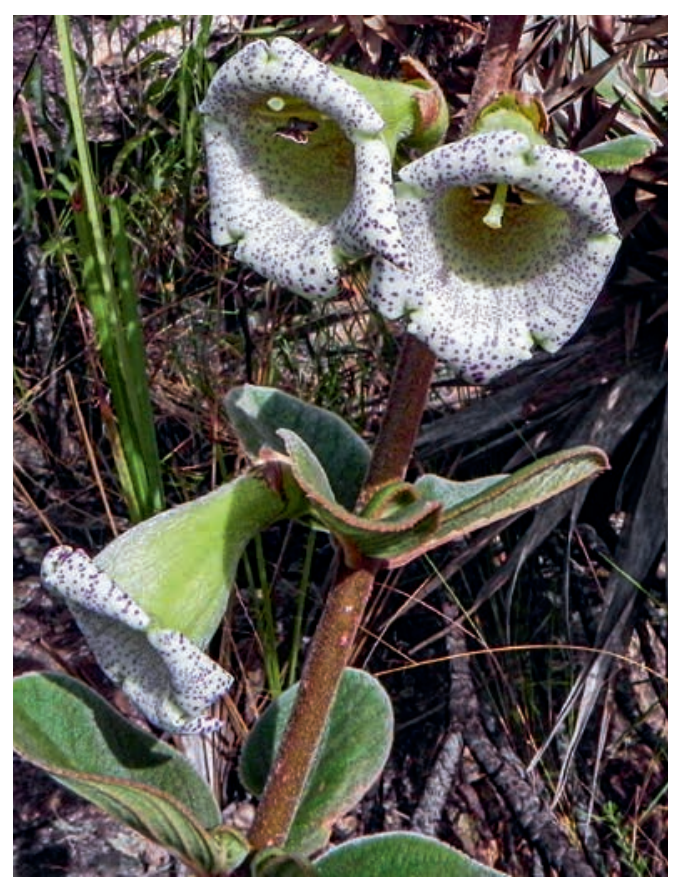

Abb. 8: Paliavana sericiflora in Brasilien (Minas Gerais, nahe Diamantina). Obwohl die Blüten Fledermausblüten sehr ähnlich sehen, werden sie von Kolibris bestäubt. (Foto: M. Регхото) 
scheinlich fledermausblütigen Arten konnten in die molekulare Analyse einbezogen werden.

Von besonderem Interesse ist das Artenpaar Paliavana prasinata und P. sericiflora. Erstere zeigt klar das Bestäubungssyndrom einer Fledermausblüte. Tatsächlich konnte hier Bestäubung durch die Fledermäuse Glossophaga soricina und Anoura caudifer dokumentiert werden (SANMARTINGajardo \& Sazima 2005b). Bei P. sericiflora weisen die Blüten chiropterophile (weit-glockige Krone, trüb-purpurne Fleckung) und schwach ornithophile Züge auf. Diese Art befindet sich anscheinend am Übergang von der Vogel- zur Fledermausblütigkeit (oder umgekehrt?). Man würde erwarten, dass hier sowohl Fledermäuse als auch Vögel als Bestäuber in Erscheinung treten. Interessanterweise ist das aber nicht der Fall: Die Blüten werden nur von Kolibris besucht (SANMartin-Gajardo \& Sazima 2005b).

Bei den schmetterlingsbestäubten Blüten muss man unterscheiden zwischen der Bestäubung durch Tagfalter (Psychophilie) und durch Nacht-

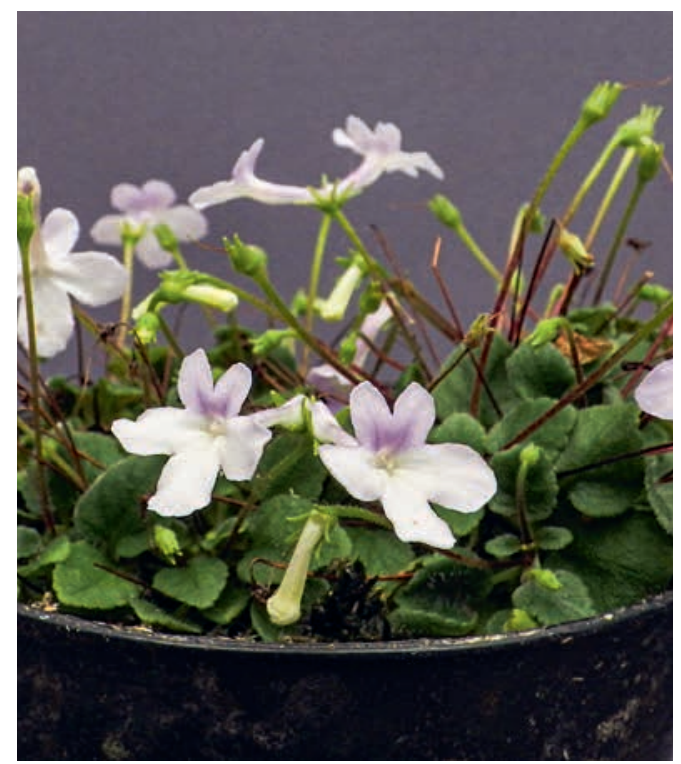

Abb. 9: Sinningia pusilla, kultivierte Pflanzen in Brasilien (Rio des Janeiro, Nahe Imné). Die dünne Kronröhre, der flache, ausgebreitete Saum und die fast waagerechte Orientierung der winzigen, 5-6 mm (!) langen Blüten deuten auf Bestäubung durch kleine Tagfalter hin. (Foto: M. Регхото) falter (Sphingophilie). Bei Sinningia sind beide Formen vertreten, aber es gibt bis dato keine Beobachtungen hierzu. Den Blütenmerkmalen zufolge (hell-lila, also eine nicht weiße Farbe; lange, enge Kronröhre; verengter Schlund, großer Saum bzw. Unterlippe in mehr oder weniger waagerechter Orientierung, der als Lande- und Sitzplatz für langrüsselige, saugende Insekten dienen kann) dürften die zwergblütigen Arten S. pusilla (Abb. 9) und S. muscicola psychophil sein.

Hingegen weist S. tubiflora (Abb. 1 F, 9) geradezu lehrbuchhaft das Syndrom der Sphingophilie auf. Die Blüten sind weiß, lang- und engröhrig und verströmen in den Abendstunden einen süßlichen oder zitronenartigen Duft.

Überblickt man die Blütenvielfalt des Sinningia-Paliavana-Vanhouttea-Komplexes (Sinningia s. 1.) im Zusammenhang mit der phylogenetischen Differenzierung, so ergibt sich ein sehr komplexes Bild. In Abb. 13 ist dieser Zusammenhang in Form eines Rades dargestellt, in dessen Inneren der molekulare Stammbaum und an der Peripherie

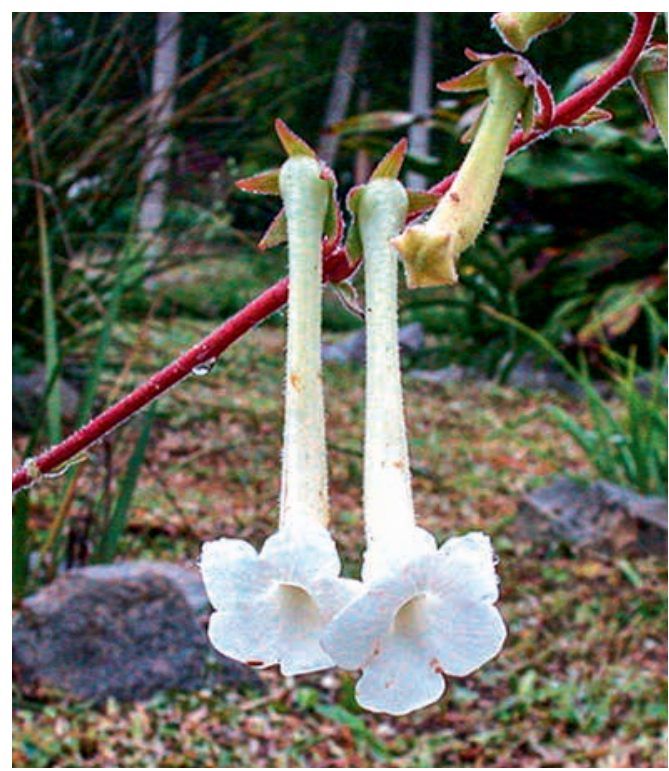

Abb. 10: Sinningia tubiflora, kultivierte Pflanze in Brasilien, Wildherkunft unbekannt. Das Syndrom der Nachtfalterblütigkeit (Sphingophilie) ist nur bei dieser einen Art, dafür aber in lehrbuchhafter Weise, repräsentiert. Vgl. dazu auch Abb. 2 F. (Foto: M. Реiхото) 
die Arten bzw. ihre Blüten stehen. Auf den ersten Blick ist eindrucksvoll zu sehen, wie oft rote, langröhrige und klar vogelbestäubte Blüten im Laufe der Evolution entstanden sind. Die molekularphylogenetische Rekonstruktion und zeitliche Interpretation des Stammbaums der neotropischen Gesneriaceen (Serrano-Serrano et al. 2007) zeigt, dass die Bienen- bzw. Insektenbestäubung der ursprüngliche Bestäubungstyp war. Aus ihm haben sich die vogelblütigen Formen entwickelt. Das Auftreten ornithophiler Blüten fällt mit dem Auftreten der Kolibris in Südamerika vor etwa 20-25 Mio. Jahren zusammen. Die neue Bestäubungsform entwickelte sich unabhängig in verschiedenen Gruppen der neotropischen Gesneriaceen, darunter auch im Sinningia-Paliavana-Vanhouttea-Komplex bzw. in dessen Vorläufer. Die Innovation der Kolibribestäubung führte zu einer doppelt so hohen Artbildungssrate wie bei der Insektenbestäubung. Die bisherige Annahme, dass mit der Vogelbestäubung ein Endpunkt in der Entwicklung erreicht war, wird durch die genannte Studie ebenfalls in Frage gestellt. Offenbar hat in der Evolution mehrmals eine Rückkehr zur Bienen- bzw. Insektenbestäubung stattgefunden.

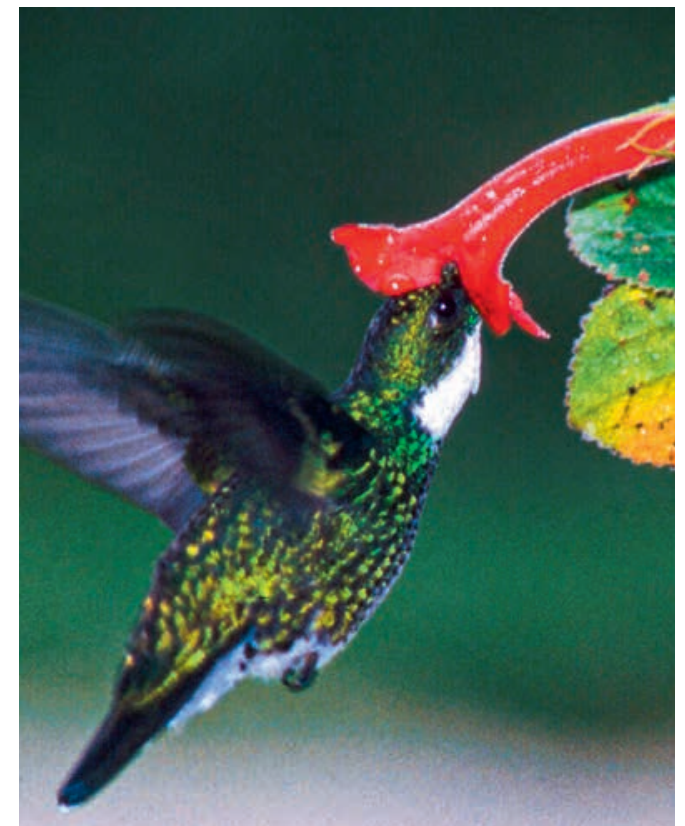

Abb. 11: Die markantesten Bestäuber von Sinningia sind Vögel (Kolibris). Hier ein Weißkehlkolibri (Leucochloris albicollis) an der Blüte von Vanhouttea hilariana in Brasilien (Minas Gerais, Parque Estadual do Ibitipoca).

(Foto: I. SanMartin-Gajardo)

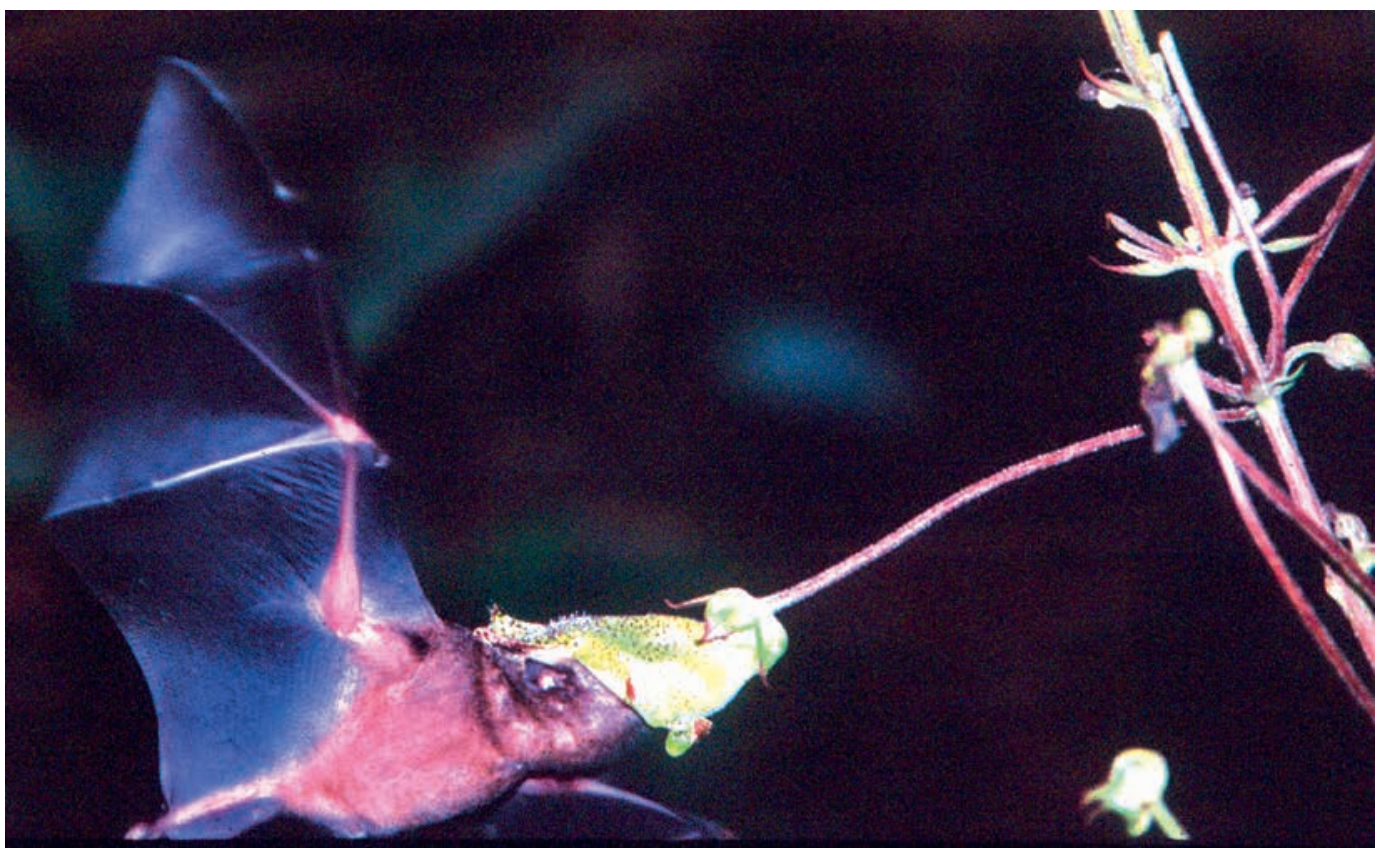

Abb. 12: Die Fledermaus Anoura caudifer an einer Blüte von Sinningia brasiliensis in Brasilien (Espírito Santo, Santa Tereza). (Foto: I. SanMartin-Gajardo) 


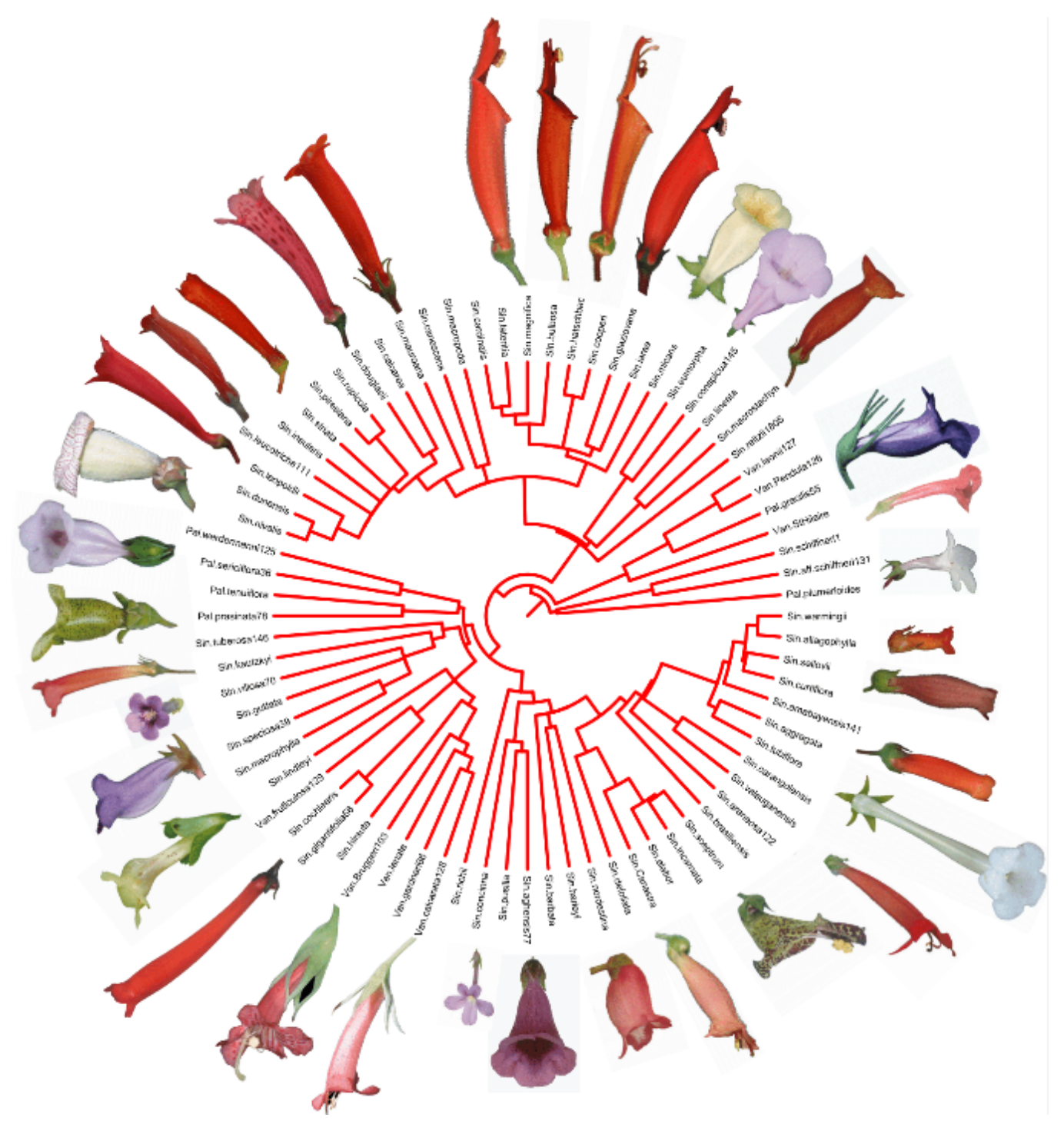

Abb. 13: Phylogenie und parallele Entstehung verschiedener Bestäubungssyndrome bei Sinningia s. 1. Erläuterungen im Text. (Graphik: M. Perret)

So muss man zum Beispiel annehmen, dass die Bienenblüte von Sinningia canastrensis aller Wahrscheinlichkeit nach aus einer kolibribestäubten Ahnenform entstanden ist.

Die weniger häufige Fledermausbestäubung ist eine deutlich jüngere Innovation, die vor 5-10 Mio. Jahren in Erscheinung trat. Fledermausblüten sind bei Sinningia s. s. einmal, beim gesamten Sinningia-Paliavana-Vanhouttea-Komplex mindestens dreimal voneinander unabhängig entstanden. Bestäubung durch Nachtfalter (Sphingophilie) ist nur einmal, und zwar bei $S$. tubiflora, entstanden, interessanterweise in einer sonst vogelblütigen Verwandtschaftsgruppe.

Es ist hier nicht der Raum, um das „Phylogenetische Rad“ in allen Details zu besprechen, aber es steckt offensichtlich voller Überraschungen und unerwarteter Details. Zusammenfassend lässt 
sich feststellen, dass die außerordentliche Blütenvielfalt des Sinningia-Komplexes durch häufige evolutionäre Wechsel der Bestäubungsform, inklusive Rückkehr zu ursprünglichen Formen der Insektenbestäubung, bedingt ist.

Das „Phylogenetische Rad“ zeigt klar auf, dass eine Verwandtschaftsgruppe, sei es eine Gattung, ein Teil einer Gattung oder in anderen Fällen eine übergeordnete Einheit, nicht auf Grund von Blütenähnlichkeiten definiert werden kann. Die Blüten sind - in relativ rascher Anpassung an wechselnde Bestäuber und Bestäubergruppen zu plastisch, um als verlässlicher Verwandtschaftszeiger gelten zu können.

Diese Erkenntnis ist keineswegs neu, sondern wurde bei den neotropischen Gesneriaceen schon vor Jahrzehnten vorweggenommen. Es war der deutsch-amerikanische Biologe Hans Wiehler (1930-2003), der in den 1970er und 1980er Jahren gegen den Strom der damaligen Systematik schwamm und die These verfocht, dass blütenmorphologische Ähnlichkeiten nicht unbedingt Ausdruck phylogenetischer Verwandtschaft sind, und umgekehrt, dass in einer Gruppe von verwandten Arten (z. B. Gattung) sehr unterschiedliche Blütenformen vertreten sein können (Wiehler 1983 und darin zitierte, frühere Arbeiten). Sein Credo „Pollination syndromes don't make genera" hat sich durch die heutige, molekular-basierte Systematik unzählige Male bestätigt. Nicht alles, was Wiehler auf den Kopf gestellt hat, war richtig, aber er war zweifellos auf dem richtigen Weg.

\section{Dank}

Zur Illustration des vorliegenden Überblicks haben neben den Autoren folgende Personen Fotos zur Verfügung gestellt: Ivonne SAnMartin-GAJARdo (Brasilien, São Paulo, Campinas/Rio Claro), Mauro Peixoto (Brasilien, São Paulo, Mogi das Cruzes), Günter Gerlach (München), und Stefan Vogel $\dagger$ (Wien). Ihnen allen sei herzlich gedankt.

\section{Literatur}

Barthlotт, W. 1990: Geschichte des Botanischen Gartens der Universität Bonn. - Veröff. Stadtarch. Bonn 48: 41-56.

Boggan, J. K. 1996: Fragrant flowers in Gesneriaceae. - Gloxinian 46: 17-27.

Martel, C., Gerlach, G., Ayasse, M. \& Milet-PinheiRO, P. 2019: Pollination ecology of the Neotropical gesneriad Gloxinia perennis: chemical composition and temporal fluctuation of floral perfume. - Plant Biol. 21: 723-731.

Perret, M., Chautems, A., Spichiger, R., Peixoto, M. \& Savolainen, V. 2001: Nectar sugar concentration in relation to pollination syndromes in Sinningieae (Gesneriaceae). - Ann. Bot. 87: 267-273.

Potgieter, C. J. \& Edwards, E. J. 2005: The Stenobasipteron wiedemannii (Diptera, Nemestrinidae) pollination guild in eastern southern Africa. - Ann. Missouri Bot. Gard. 92: 254-267.

SanMartin-Gajardo, I. \& Sazima, M. 2004: Non-Euglossine bees also function as pollinators of Sinningia species (Gesneriaceae) in Southeastern Brazil. - Plant Biol. 6: 506-512.

SanMartin-Gajardo, I. \& Sazima, M. 2005a: Species of Vanhouttea Lem. and Sinningia Nees (Gesneriaceae) pollinated by hummingbirds: interactions related with plant habitat and nectar. - Rev. Brasil. Bot. 28: 441-450.

SanMartin-Gajardo, I. \& Sazima, M. 2005b. Chiropterophily in Sinningieae (Gesneriaceae): Sinningia brasiliensis and Paliavana prasinata are bat-pollinated, but $P$. sericiflora is not. Not yet? - Ann. Bot. 95: 1097-1103.

Serrano-Serrano M. L., Rolland, J., Clark, J. L., Salamin, N. \& Perret, M. 2017: Hummingbird pollination and the diversification of angiosperms: An old and successful association in Gesneriaceae. - Proc. R. Soc. B. 284, 1852.

Vogel, S. 1966: Parfümsammelnde Bienen als Bestäuber von Orchideen und Gloxinia. - Österr. Bot. Z. 113: 302-361.

Weber, A. \& Gerlach, G. 2018: Die großen wissenschaftlichen Leistungen von Stefan Vogel. Teil 4. Die „Parfümblumen“ und ihre prächtigen Bestäuber. - Palmengarten 81: 133-148.

Wiehler, H., 1983: A synopsis of Neotropical Gesneriaceae. Selbyana 6: 1-219.

\section{Anschrift der Autoren}

Dr. Alain Chautems, Dr. Mathieu Perret, Conservatoire et Jardin botaniques de la Ville de Genève, Schweiz. E-Mail: alain.chautems@ville-ge.ch, mathieu.perret@ville-ge.ch

Prof. i.R. Dr. Anton Weber, Department für Botanik und Biodiversitätsforschung, Universität Wien, Rennweg 14, A-1030 Wien. E-Mail: anton.weber@univie.ac.at 